\title{
Outcome of Frail Do-Not-Intubate Subjects With End-Stage Chronic Respiratory Failure and Their Opinion of Noninvasive Ventilation to Reverse Hypercapnic Coma
}

\author{
Malcolm Lemyze, Gaël De Palleja, Aurélie Guiot, Quentin Bury, Marie Jonard, Maxime Granier, \\ Didier Thevenin, and Jihad Mallat
}

\begin{abstract}
BACKGROUND: The use of noninvasive ventilation (NIV) in the emergency setting to reverse hypercapnic coma in frail patients with end-stage chronic respiratory failure and do-not-intubate orders remains a questionable issue given the poor outcome of this vulnerable population. We aimed to answer this issue by assessing not only subjects' outcome with NIV but also subjects' point of view regarding NIV for this indication. METHODS: A prospective observational case-control study was conducted in 3 French tertiary care hospitals during a 2-y period. Forty-three individuals who were comatose (with $\mathrm{pH}<7.25$ and $\mathrm{P}_{\mathrm{aCO}_{2}}>100 \mathrm{~mm} \mathrm{Hg}$ at admission) were compared with 43 subjects who were not comatose and who were treated with NIV for acute hypercapnic respiratory failure. NIV was applied by using the same protocol in both groups. They all had a do-notintubate order and were considered vulnerable individuals with end-stage chronic respiratory failure according to well-validated scores. RESULTS: NIV yielded similar outcomes in the 2 groups regarding in-hospital mortality $(n=12[28 \%]$ vs $n=12[28 \%]$ in the noncomatose controls, $P>.99)$ and 6-month survival $(n=28[65 \%]$ vs $n=22[51 \%]$ in the noncomatose controls, $P=.31)$. Despite poor quality of life scores $(21.5 \pm 10 \mathrm{vs} 31 \pm 6$ in the awakened controls, $P=.056)$ as assessed by using the VQ11 questionnaire 6 months to $1 \mathrm{y}$ after hospital discharge, a large majority of the survivors $(n=23$ [85\%]) would be willing to receive NIV again if a new episode of acute hypercapnic respiratory failure occurs. CONCLUSIONS: In the frailest subjects with supposed end-stage chronic respiratory failure that justifies treatment limitation decisions, it is worth trying NIV when acute hypercapnic respiratory failure occurs, even in the case of extreme respiratory acidosis with hypercapnic coma at admission. Key words: respiratory insufficiency; noninvasive ventilation; do-not-intubate order; do-not-resuscitate orders; COPD; obesity hypoventilation syndrome. [Respir Care 2019;64(9):1023-1030. ㅇ 2019 Daedalus Enterprises]
\end{abstract}

\section{Introduction}

Despite the cumulative evidence that supports noninvasive ventilation (NIV) for acute hypercapnic respiratory

\footnotetext{
Dr Lemyze, Dr De Palleja, Dr Jonard, Dr Thevenin, and Dr Mallat are affiliated with the Department of Respiratory and Critical Care Medicine, Schaffner Hospital, Lens, France. Drs Lemyze, Jonard, Granier, Thevenin, and Mallat are affiliated with the Intensive Care Unit, Arras Hospital, Arras, France. Dr De Palleja is affiliated with the Respiratory Intermediate Care Unit, Schaffner Hospital, Lens, France. Dr Guiot is affiliated with the Department of Cardiology, Bois Bernard Hospital, Rouvroy, France. Dr Bury is affiliated with the Respiratory Intermediate Care Unit, Beuvry Hospital, Béthune, France.
}

failure, ${ }^{1}$ NIV may be considered questionable for vulnerable patients with do-not-intubate orders, especially when hypercapnic coma occurs. However, NIV can relieve dys-

\footnotetext{
Supplementary material related to this paper is available at http:// www.rcjournal.com.

The authors have disclosed no conflicts of interest.

Correspondence: Malcolm Lemyze, MD, Department of Respiratory and Critical Care Medicine, Schaffner Hospital, 99 route de la Bassée, 62300 Lens, France. E-mail: malcolmlemyze@yahoo.fr.
}

DOI: $10.4187 /$ respcare. 06346 


\section{DNI SubJeCts' OPINIONS OF NIV}

pnea, improve comfort, and serve as a rescue therapy that can facilitate hospital discharge for some patients who are terminally ill and want to go home. ${ }^{2-5}$ According to the large multi-center observational study conducted by Azoulay et al, ${ }^{6}$ survivors of an episode of acute respiratory

\section{See the Related Editorial on Page 1169}

failure treated with NIV as a ceiling of care did not exhibit a lower health-related quality of life or higher symptoms of depression, anxiety, and post-ICU burden compared with baseline and with subjects without any treatmentlimitation decisions. Yet, implementing a ventilatory support for frail patients with an underlying terminal disease, such as end-stage chronic respiratory failure, may be regarded as an excessive, unreasonable escalation that merely prolongs the dying process. ${ }^{7-9}$ The latter seems reasonable when these already frail patients present with reduced consciousness or coma. Results of two studies indicate that impaired consciousness at admission was an independent predictor of NIV failure and in-hospital mortality in subjects with a do-not-intubate order and who were in acute hypercapnic respiratory failure. ${ }^{4,5}$ However, there are limited data to support any of these contradictory management strategies for frail patients with severe hypercapnia. Severe hypercapnic coma prevents the patient from sharing his or her will regarding end of life, from giving informed consent, and, ultimately, from participating in the decision-making process. When there is no clear advance directive from the patient regarding NIV, the clinician and/or relatives have to choose between 2 irreconcilable points of view. NIV can be considered a costly futile therapeutic attempt for a frail patient who is comatose and seemingly approaching the end of life; whereas NIV could be proposed as the last noninvasive treatment that could possibly facilitate the awakening and recovery of the patient. Because few patients have already discussed their end-of-life wishes, ${ }^{10}$ this situation is unfortunately very common in clinical practice. In this particular scenario, the attending physicians risk inappropriate therapeutics versus certain mortality without timely intervention. What is needed to try to answer this difficult question is the patient's point of view rather than just reporting in-hospital mortality.

In a prospective observational study, we showed that switching the interface to a total face mask enabled the pursuit of NIV when face mask-delivered NIV had already failed in subjects with a do-not-intubate order and in acute hypercapnic respiratory failure. ${ }^{11}$ This strategy enabled two thirds of the subjects to survive to hospital discharge, while predicted mortality was high. ${ }^{11}$ Since this first positive experience, we have applied this protocol to many hypercapnic patients with end-stage chronic respi-

\section{QUICK LOOK}

\section{Current knowledge}

Noninvasive ventilation (NIV) may be considered a needless therapeutic intervention in frail patients with end-stage chronic respiratory failure and do-not-intubate orders, especially in cases of hypercapnic coma. When comatose, patients with a do-not-intubate order cannot participate in the decision-making process regarding NIV initiation and their end-of-life care. Clinicians risk of unreasonable obstinacy in therapeutics, by uselessly prolonging the dying process with NIV or not providing appropriate care if NIV is prematurely stopped.

\section{What this paper contributes to our knowledge?}

In a population of vulnerable individuals diagnosed with end-stage respiratory failure and treated by NIV for hypercapnic coma, $70 \%$ survived to hospital discharge and half survived $>6$ months, with similar outcomes to controls who were not comatose and also treated with NIV for the same indication. Despite poor quality of life, $85 \%$ of the survivors would be willing to receive NIV again in the case of a new episode of acute-onchronic respiratory failure.

ratory failure, and, in many cases, we have successfully pushed the boundaries of what can be done with NIV. Herein, we presented the results of this strategy in subjects with the most-severe cases of hypercapnic encephalopathy compared with subjects not comatose and with a do-notintubate order and with acute hypercapnic respiratory failure We reported their outcome and their opinion regarding NIV for this indication.

\section{Methods}

This prospective observational case-control study was approved by our local institutional ethics committee (Comité d'éthique du Centre Hospitalier du Dr Shaffner, Lens, France) with the approval number 150601. It was conducted over a 2 -y period in 2 ICUs and 2 respiratory intermediate care units from 3 tertiary care hospitals in the north of France. Signed informed consent was obtained from all the subjects or their next of kin.

Forty-three consecutive subjects were classified as having a do-not-intubate order and were being treated by NIV for hypercapnic coma at admission. Clinical criteria that defined acute hypercapnic respiratory failure included tachypnea ( $>24$ breaths $/ \mathrm{min}$ ), signs of respiratory distress with increased work of breathing, Hoover's sign, acces- 


\section{DNI SuBJECTS' OPINIONS OF NIV}

sory muscle use, and paradoxical abdominal motion. ${ }^{1,11}$ Blood gas criteria for acute hypercapnic respiratory failure included at least a $\mathrm{pH}$ of $<7.35$ and $\mathrm{P}_{\mathrm{aCO}_{2}}$ of $>45 \mathrm{~mm} \mathrm{Hg}$. ${ }^{1,11}$ The severity of hypercapnic coma was classified according to the scale of Kelly and Matthay ${ }^{12}$ as follows: grade 1, alert, follows complex 3-step commands; grade 2, alert, follows simple commands; grade 3 , lethargic but arousable and follows simple commands; grade 4, stuporous, only intermittently follows simple commands; grade 5, comatose with the brain stem intact; grade 6, comatose with brain stem dysfunction.

The definition of hypercapnic coma was a composite of the clinical criteria of acute hypercapnic respiratory failure with the scale of Kelly and Matthay ${ }^{12} \geq 5$ combined with a $\mathrm{pH}$ of $<7.25$ and $\mathrm{P}_{\mathrm{aCO}_{2}}$ of $>100 \mathrm{~mm} \mathrm{Hg}$. Swallowing disorders and copious bronchial secretions were evaluated by the attending clinician on a case-by-case basis without these being considered as absolute contraindications to NIV. The subjects who were comatose at admission were compared with a cohort of subjects with a do-not-intubate order and who were in acute hypercapnic respiratory failure without severe hypercapnic coma syndrome (the Kelly and Matthay ${ }^{12}$ scale $\leq 4$ ) who were managed by NIV during the same time period.

\section{Do-Not-Intubate Order Status}

A limitation of invasive life support is commonly discussed in frail individuals with end-stage chronic respiratory disease because such patients are considered poor candidates for intubation and prolonged mechanical ventilation. ${ }^{8,13}$ According to good ethical practice and standards of care, the do-not-intubate order status of the subjects resulted from a consensual decision-making process by either the patient or the appointed trusted person whenever possible; this has been extensively described in previously published articles. ${ }^{11,14}$ Briefly, all patients admitted for acute hypercapnic respiratory failure were classified as with or without a do-not-intubate order. This decision was made by the patient whenever possible. When it could not be made by the patient, the decision was made by the trusted person mandated by the patient or by a multidisciplinary team, including physicians and nurses who cared for the patient. Clinicians involved in the decision-making process included at least an intensivist and either a pulmonologist or an emergency care practitioner, and none participated in the present study. The subjects were classified as with a do-not-intubate order when their physical disability and their underlying debilitating conditions made them poor candidates for intubation. The subject's family received clear and thorough information, and all efforts were made to facilitate understanding and adherence to the medical decisions. ${ }^{11,14}$

\section{NIV Protocol}

Once the diagnosis of acute hypercapnic respiratory failure was made, NIV was started by using a nonvented full face mask (PerformaTrak, Philips Respironics, Murrysville, Pennsylvania) and a turbine-driven portable ventilator (BiPAP Vision, Philips Respironics) connected via its single-limb circuit. The NIV circuit was equipped with a precalibrated intentional air leak that was positioned as close as possible to the subject. The subject was comfortably placed in the sitting position, either in the bed or in a chair, and received explanations regarding the NIV technique and the interface. The spontaneous-timed mode of the BiPAP Vision ventilator was used with adaptive triggering and cycling. The ventilator settings were left to the discretion of the physician in charge. The criteria used to define NIV success and NIV failure are fully described in previous publications. ${ }^{11,14}$ When NIV failed to reverse the acute respiratory failure episode, compassionate care with terminal sedation was instituted and the family was invited to remain with the dying patient until the end.

\section{Evaluation Criteria}

Anthropometric data, smoking history, causes of acute hypercapnic respiratory failure, the underlying lung disease and its severity according to the Medical Research Council dyspnea score, ${ }^{15}$ the main ventilator settings, and the duration of NIV were recorded. Exacerbation of chronic respiratory disease refers to an acute hypercapnic respiratory failure episode without any clearly identified cause. Pre-admission functional health status was assessed by using the Charlson comorbidity index, ${ }^{16}$ the clinical frailty score as detailed in the supplementary material (see the supplementary materials at http://www.rcjournal.com), ${ }^{17}$ and the Knaus index. ${ }^{18}$ In the latter index, class $\mathrm{C}$ refers to patients with a severe chronic life-limiting illness but not a fully incapacitating condition, whereas class D refers to patients who are usually bedridden, unable to work, or are institutionalized and dependent on others for their activities of daily living. The Simplified Acute Physiologic Score II and the sequential organ failure assessment score were used to assess the risk of death and the subjects' severity at admission. ${ }^{19,20}$

A short survey was conducted by a telephone call to evaluate how the NIV strategy was judged by the subjects who survived, if they were capable of answering the queries. The short survey was performed by our medical secretary 6 months after each subject's hospital discharge. It should be stressed that the interviewer was not involved in the data analysis of the present study. The study took place in the most socioeconomically disadvantaged part of France. ${ }^{21}$ Given the poor educational level of our subjects, we chose the simplest, shortest, and most understandable 


\section{DNI SuBJECTs' OPINIONS OF NIV}

questionnaire, the VQ11, to accurately assess our subjects' quality of life. As detailed in the online supplementary material, the VQ11 is a health-related quality-of-life questionnaire validated in patients with chronic respiratory diseases and takes into account physical, emotional, and social well-being. It includes 11 questions evaluated with a 5-point Likert-type scale: 1, not at all; 2, a little; 3, average; 4, a lot; and 5, extremely. ${ }^{22,23}$ A VQ11 score $\geq 22$ reflects a poor quality of life. At the end of the questionnaire, the subject had to answer a simple question: "would you be willing to receive NIV again in the case of a new episode of acute respiratory failure or would you prefer palliative care only?"

\section{Statistical Analysis}

Data are expressed as mean \pm SD when normally distributed or as median (25-75\% interquartile range) when non-normally distributed. The normality of data distribution was assessed by using the Kolmogorov-Smirnov test. Comparisons of values the between different groups of subjects were performed by using the 2-tailed Student $t$ test or Mann-Whitney test, as appropriate. Analysis of categorical data were performed by using the chi-square and Fisher exact tests. Cox proportional hazards regression multivariable analysis (entry $P<.15$ ) was used to identify significant independent predictors that were associated with 6-month mortality in the subjects who were comatose. Proportional hazards assumption was assessed by using the Schoenfeld test. The potential problem of co-linearity was evaluated before running the analysis. Kaplan-Meier curves for cumulative survival during the 6-month period were constructed and compared with the use of the logrank test. Statistical analysis was performed by using Stata 14.0 (StataCorp, College Station, Texas). $P<.05$ was considered statistically significant. All reported $P$ values are 2-sided.

\section{Results}

We studied 86 frail subjects in acute hypercapnic respiratory failure who had do-not-intubate orders. As shown in Table 1, all the subjects exhibited high comorbidity scores, severe respiratory disease, poor functional status, and severe respiratory acidosis at admission. In-hospital mortality was $28 \%$, and 50 subjects $(58 \%)$ survived $>6$ months. Of these, 9 died, 10 were unable to answer the queries, 4 were lost to follow-up, and 27 were consultable at the time of the interview. More than half $(n=16$ [60\%]) could be considered as having a poor quality of life, as evidenced by a VQ11 score of $\geq 22$. Among survivors interviewed 6 to 12 months after hospital discharge, 23 subjects $(85 \%)$ were willing to receive NIV again in case of a new episode of acute hypercapnic respiratory failure.
The subjects who were comatose $(n=43)$ and the controls $(n=43)$ did not differ significantly in age, sex, body mass index, nutritional status, history of respiratory disease, comorbidity score, smoking habit, and $\mathrm{P}_{\mathrm{aO}_{2}} / \mathrm{F}_{\mathrm{IO}_{2}}$. The subjects who were comatose had lower $\mathrm{pH}$ and higher encephalopathy scores, $\mathrm{P}_{\mathrm{aCO}}$, bicarbonate level, and severity scores at admission compared with the subjects who were awake at admission. More exacerbations of chronic respiratory disease and less pneumonia were diagnosed in the subjects who were comatose than in the control group (Table 1). NIV yielded similar outcomes in the 2 groups regarding in-hospital mortality, 6-month survival (Fig. 1), and quality of life as assessed by the VQ11 questionnaire (Table 2). The subjects' survival according to the initial severity of the acute hypercapnic respiratory failure episode at admission are shown in Figure 1. The factors independently associated with 6-month mortality included age, presence of pneumonia, and pre-admission functional health status as assessed by the Knaus index before hospitalization (Table 3). Regarding quality of life, there was no statistically significant difference in the median (interquartile range) VQ11 levels between the subjects who were comatose and who survived $>6$ months and awakened controls $(21[17-27]$ vs 31 [25-31], $P=.056)$, respectively.

\section{Discussion}

The main findings of the present study were the following: (1) NIV is usually successful in frail subjects with acute on chronic respiratory failure and who had a do-notintubate order, even in the case of hypercapnic coma with extremely severe respiratory acidosis at admission, (2) more than half of these fragile subjects survived $>6$ months after acute hypercapnic respiratory failure, and (3) despite poor quality of life, most of the subjects would choose to be placed on NIV again if a new episode of acute hypercapnic respiratory failure occurred.

Scala et $\mathrm{al}^{24}$ reported outcomes in subjects with COPD who were hypercapnic according to the severity of their encephalopathy score when treated with full face-mask NIV as part of full-code management strategy. Those with a higher degree of encephalopathy experienced higher NIV failure and in-hospital mortality rates compared with those with a lower encephalopathy score at admission. ${ }^{24}$ Interestingly, the reported in-hospital mortality of patients with COPD, hypercapnic, and comatose who are treated with NIV are consistently lower than those with acute hypercapnic respiratory failure and who are intubated without an NIV trial. ${ }^{24-26}$ In the largest series, by Díaz, et al ${ }^{26}$ the survival rate reached $74 \%$ among 95 subjects with COPD treated by NIV for hypercapnic coma. Patients who are hypercapnic and with a do-not-intubate order usually experience poorer outcomes compared with those without 
Table 1. Subject Characteristics at Admission

\begin{tabular}{|c|c|c|c|c|}
\hline Characteristic & $\begin{array}{l}\text { Overall } \\
\text { Population } \\
(N=86)\end{array}$ & $\begin{array}{l}\text { Subjects Who } \\
\text { Were Comatose } \\
\quad(n=43)\end{array}$ & $\begin{array}{c}\text { Subjects Who } \\
\text { Were Not } \\
\text { Comatose }(n=43)\end{array}$ & $P$ \\
\hline Age, median (IQR) y & $74(62-82)$ & $73(61-82)$ & $76(62-83)$ & .69 \\
\hline Male, $n(\%)$ & $42(49)$ & $21(49)$ & $21(49)$ & $>.99$ \\
\hline Weight, median (IQR) kg & $82(62-107)$ & $81.5(65-114)$ & $83(64-105)$ & .80 \\
\hline BMI, median (IQR) kg/m² & $30(22-45)$ & $31(22-45)$ & $30(22-45)$ & .61 \\
\hline Albumin, mean $\pm \mathrm{SD} g / \mathrm{L}$ & $29 \pm 5$ & $30.4 \pm 6.4$ & $28.5 \pm 4.4$ & .14 \\
\hline SAPS II, mean \pm SD & $42 \pm 9$ & $46 \pm 8$ & $37 \pm 8$ & $<.001$ \\
\hline Charlson comorbidity index score, median (IQR) & $4(3-5)$ & $4(3-5)$ & $4(3-5)$ & .80 \\
\hline MRC dyspnea score, median (IQR) & $5(5-5)$ & $5(5-5)$ & $5(5-5)$ & .57 \\
\hline Clinical frailty score, median (IQR) & $6(5-7)$ & $6(5-7)$ & $6(5-6)$ & .32 \\
\hline Knaus index C, $n(\%)$ & $33(38)$ & $17(39)$ & $16(37)$ & .82 \\
\hline Knaus index $\mathrm{D}, n(\%)$ & $52(60)$ & $26(60)$ & $26(60)$ & $>.99$ \\
\hline Cancer, $n(\%)$ & $10(11.6)$ & $5(11.6)$ & $5(11.6)$ & $>.99$ \\
\hline \multicolumn{5}{|l|}{ Underlying lung disease, $n(\%)$} \\
\hline COPD & $46(53.5)$ & $23(53)$ & $23(53)$ & $>.99$ \\
\hline Obesity-hypoventilation syndrome & $17(19.7)$ & $8(18.6)$ & $9(21)$ & .80 \\
\hline Kyphoscoliosis & $17(19.7)$ & $9(21)$ & $8(18.6)$ & .80 \\
\hline Interstitial lung disease & $3(3.5)$ & $1(2.3)$ & $2(4.6)$ & .17 \\
\hline Other & $3(3.5)$ & $2(4.6)$ & $1(2.3)$ & .17 \\
\hline \multicolumn{5}{|l|}{ Cause of acute respiratory failure, $n(\%)$} \\
\hline Exacerbation of chronic disease & $44(51)$ & $27(62.8)$ & $17(39.5)$ & .032 \\
\hline Pneumonia & $22(25.6)$ & $6(14)$ & $16(37)$ & .01 \\
\hline Congestive heart failure & $12(14)$ & $5(11.6)$ & $7(16.3)$ & .53 \\
\hline Sepsis & $4(4.6)$ & $2(4.6)$ & $2(4.6)$ & $>.99$ \\
\hline Other & $4(4.6)$ & $3(7)$ & $1(2.3)$ & .60 \\
\hline \multicolumn{5}{|l|}{ Clinical parameters } \\
\hline Frequency, mean \pm SD breaths/min & $28 \pm 8$ & $29 \pm 9$ & $27 \pm 7$ & .37 \\
\hline Encephalopathy score, median (IQR) & $4(3-5)$ & $5(5-5)$ & $3(2-4)$ & $<.001$ \\
\hline $\mathrm{SABP}$ score, mean $\pm \mathrm{SD}$ mm Hg & $138 \pm 27$ & $145 \pm 32$ & $130 \pm 18$ & .008 \\
\hline \multicolumn{5}{|l|}{ Arterial blood gases } \\
\hline $\mathrm{pH}$, median (IQR) & $7.21(7.13-7.27)$ & $7.15(7.06-7.21)$ & $7.27(7.23-7.32)$ & $<.001$ \\
\hline $\mathrm{P}_{\mathrm{aCO}_{2}}$, median (IQR) mm Hg & $98(74-113)$ & $113(103-141)$ & $74(65-81)$ & $<.001$ \\
\hline $\mathrm{P}_{\mathrm{aO}_{2}} / \mathrm{F}_{\mathrm{IO}_{2}}$, median (IQR) & $217(164-270)$ & $217(150-283)$ & $217(183-263)$ & .73 \\
\hline $\mathrm{F}_{\mathrm{IO}_{2}}$, median (IQR) \% & $40(30-50)$ & $40(30-50)$ & $35(30-50)$ & .19 \\
\hline $\mathrm{HCO}_{3}{ }^{-}$, mean $\pm \mathrm{SD} \mathrm{mmol} / \mathrm{L}$ & $36.5 \pm 7.7$ & $40.4 \pm 7.5$ & $32.5 \pm 5.7$ & $<.001$ \\
\hline EPAP, median (IQR) $\mathrm{cm} \mathrm{H}_{2} \mathrm{O}$ & $8(6-8)$ & $8(6-9)$ & $8(6-8)$ & .18 \\
\hline IPAP, median (IQR) $\mathrm{cm} \mathrm{H}_{2} \mathrm{O}$ & $18(16-20)$ & $18(17-20)$ & $16(16-19)$ & .02 \\
\hline $\begin{array}{l}\mathrm{IQR}=\text { interquartile range } \\
\text { BMI }=\text { body mass index } \\
\text { SAPS II = Simplified Acute Physiologic Score II } \\
\text { MRC = Medical Research Council dyspnea scale } \\
\text { Knaus index } C=\text { serious but not incapacitating restriction of activi } \\
\text { Knaus index } D=\text { severe restriction of activity due to disease inclu } \\
\text { SABP = systolic arterial blood pressure } \\
\text { EPAP = expiratory positive airway pressure } \\
\text { IPAP = inspiratory positive airway pressure }\end{array}$ & Iden or institutionalized s & & & \\
\hline
\end{tabular}

treatment limitations, not because aggressive invasive management provides benefits but rather as the result of patients with a do-not-intubate order being more vulnerable overall. ${ }^{8}$ In this population, NIV provides better outcomes in cases of hypercapnic exacerbation of COPD or cardiogenic pulmonary edema rather than in cases of cancer or community-acquired pneumonia. ${ }^{4-6}$
Our population included fragile patients with a high comorbidity score, poor functional status, and severe chronic respiratory failure according to well-validated scores. Their respiratory illness was considered nearly at its final stage. Thus, they had refused intubation or were deemed poor candidates for invasive life-support modalities. Moreover, the exacerbation could be considered as 


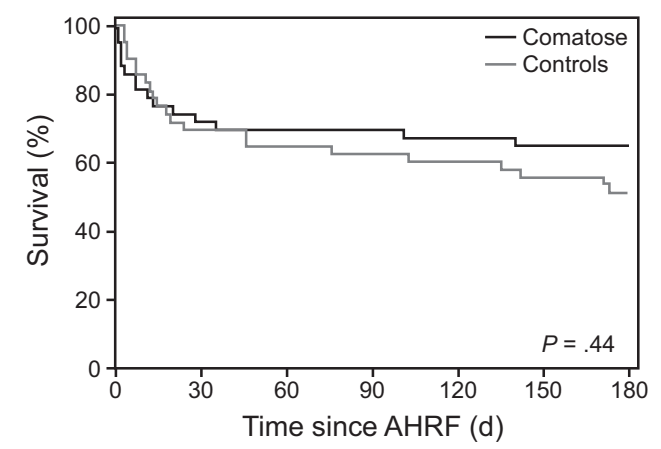

Fig. 1. Outcome of subjects according to the severity of acute hypercapnic respiratory failure (AHRF) at admission.

Table 2. Duration of NIV, Length of Stay, and Mortality

\begin{tabular}{|c|c|c|c|}
\hline Parameter & $\begin{array}{c}\text { Subjects } \\
\text { Who Were } \\
\text { Comatose } \\
(n=43)\end{array}$ & $\begin{array}{c}\text { Subjects Who } \\
\text { Were Not } \\
\text { Comatose } \\
(n=43)\end{array}$ & $P$ \\
\hline \multicolumn{4}{|l|}{ Duration of NIV } \\
\hline $\begin{array}{l}\text { In the first } 48 \mathrm{~h} \text {, median } \\
\text { (IQR) } \mathrm{h}\end{array}$ & $38(28-45)$ & $34(26-36)$ & .02 \\
\hline In the ICU, median (IQR) d & $5(3-8)$ & $6(4-8)$ & .36 \\
\hline \multicolumn{4}{|l|}{ Length of stay, median (IQR) d } \\
\hline In-hospital $(n=13)$ & $12(8-17)$ & $14(10-21)$ & .15 \\
\hline In the ICU $(n=7)$ & $7(4-12)$ & $7(4-19)$ & .60 \\
\hline In-hospital mortality $(n=24), n(\%)$ & $12(28)$ & $12(28)$ & $>.99$ \\
\hline \multicolumn{4}{|l|}{$\overline{N=86}$} \\
\hline
\end{tabular}

very severe because it was associated with extreme respiratory acidosis and complicated by an altered mental status. Despite the cumulative evidence for poor hospital outcome in these frail subjects, NIV was successful at reversing acute hypercapnic respiratory failure in most, and it allowed a majority to return home. Against all expectations, many of them survived $>6$ months, and, despite a poor quality of life, the majority of them would choose to be placed on NIV again if their situation deteriorated and a new episode of acute hypercapnic respiratory failure occurred.

One interesting hypothesis would be that carbon dioxide narcosis acts as a natural sedation that would help patients adhere to NIV. Comatose patients who survive after hospital discharge would then have no particular aversion to repeated NIV. In the present population, NIV did not uselessly prolong the end of life in fragile subjects with donot-intubate orders but rather offered these subjects a real chance of being discharged home to their family and loved ones. Several studies pointed out that both physicians and commonly used predictive models usually fail to accurately determine the time of death in the subjects with the most-severe COPD who are approaching end of life. ${ }^{27-29}$
Table 3. Predictors of 6-Month Mortality in the Subjects Who Were Comatose

\begin{tabular}{|c|c|c|}
\hline Parameter & $\operatorname{HR}(95 \% \mathrm{CI})$ & $P$ \\
\hline \multicolumn{3}{|l|}{ Univariate analysis } \\
\hline Age & $1.06(1.011-1.129)$ & .02 \\
\hline Sex & $0.90(0.326-2.484)$ & .84 \\
\hline BMI & $0.99(0.955-1.027)$ & .61 \\
\hline Albumin & $1.00(0.928-1.085)$ & .92 \\
\hline SOFA score & $1.39(0.885-2.175)$ & .15 \\
\hline SAPS II & $1.03(0.972-1.091)$ & .32 \\
\hline Charlson comorbidity score & $1.39(1.130-1.710)$ & .002 \\
\hline Clinical frailty score & $1.30(0.722-2.327)$ & .38 \\
\hline Knaus index & $3.13(0.883-11.114)$ & .08 \\
\hline Pneumonia & $3.05(0.960-9.708)$ & .059 \\
\hline $\mathrm{pH}$ & $0.09(0.00-17.138)$ & .37 \\
\hline $\mathrm{P}_{\mathrm{aCO}_{2}}$ & $1.03(1.001-1.055)$ & .045 \\
\hline $\mathrm{P}_{\mathrm{aO}_{2}} / \mathrm{F}_{\mathrm{IO}_{2}}$ & $1.00(0.991-1.004)$ & .50 \\
\hline \multicolumn{3}{|l|}{ Multivariate analysis } \\
\hline Age & $1.06(1.019-1.099)$ & .003 \\
\hline Knaus index & $3.13(1.399-7.025)$ & .006 \\
\hline Charlson comorbidity score & $1.08(0.911-1.283)$ & .37 \\
\hline Pneumonia & $2.81(1.268-6.256)$ & .01 \\
\hline $\mathrm{P}_{\mathrm{aCO}}$ & $1.00(0.986-1.012)$ & .91 \\
\hline \multicolumn{3}{|c|}{$\begin{array}{l}\mathrm{HR}=\text { hazard ratio } \\
\text { BMI = body mass index } \\
\text { SOFA = Sequential Organ Failure Assessment } \\
\text { SAPS II = Simplified Acute Physiologic Score II }\end{array}$} \\
\hline
\end{tabular}

As with COPD, chronic hypercapnic respiratory diseases have a highly variable course that is sometimes progressive, but, more often, the disease follows a chaotic path marked by attacks of acute respiratory failure, which makes their prognosis unpredictable. ${ }^{30}$ Patients' decisions regarding treatment limitations and choices can drastically change over the last months of life and even during the course of a hospitalization. ${ }^{28,31}$ All of these factors, including variable disease course and patients' choices, and the poor reliability of the prognostication model, result in very hard task for the attending clinician caring for those patients in acute hypercapnic respiratory failure.

Further research is needed to identify the predictors of poor response to NIV in this particular population of frail patients with acute on chronic respiratory failure, to avoid futile continuation of the technique when it is obvious that it will fail. Similarly, reliable predictors of NIV success are needed to avoid inappropriate NIV withdrawal in this vulnerable population, which may lead to unnecessary mortality. The perception of whether NIV would be a valuable life-sustaining treatment (rather than a burden) when faced with a supposed end-stage respiratory disease is likely to be heavily affected by the culture, religious belief, opinion of caregivers, reimbursement system, financial situations, and many other factors. Clinicians should remain humble 


\section{DNI SubJeCts' OpINIONS OF NIV}

and discuss each situation, on a case-by-case basis, with the patient whenever possible or with the family.

Several limitations of this study have to be acknowledged. Because of the prospective observational study design, selection bias cannot be ruled out. However, randomization seems inappropriate to answer this issue, apart from the frailty of these patients and their do-not-intubate order status. Indeed, it seemed unethical to us to randomize subjects between NIV versus standard treatment with no ability to intubate in either group. A study design that does not allow any invasive ventilation support in one arm would result in a better outcome for the NIV group compared with standard treatment alone. Secondary to ethical concerns, every issue we have in medicine cannot always be resolved by randomized, controlled trials, especially in the palliative care setting. ${ }^{32}$

At the time of the follow-up telephone call interview, a significant proportion of the subjects had died or were not consultable anymore because of delirium, dementia, hearing loss, or extreme frailty. We could not ensure that these individuals would have been willing to receive NIV again. One would argue that more community-acquired pneumonia was found in the control group than in the subjects who were comatose, whereas pneumonia yields the highest morbidity and/or mortality among the causes of acute respiratory failure in patients with chronic respiratory failure. ${ }^{33,34}$ However, with two thirds of the frail subjects who were comatose and had a do-not-intubate order and survived to hospital discharge, our results clearly supported the use of NIV. No significant difference could be identified between the subjects who were comatose and the controls who were not comatose regarding anthropometric data, underlying respiratory diseases, comorbidities, and functional health status. Furthermore, NIV was applied according to a strict protocol by using the same type of ventilator, circuit, same interface, and patient positioning for all the subjects in 4 different care units. Also, patients' outcome with NIV depend on the attending team's experience with the technique; our findings were those of well-trained staff with adequate patient monitoring.

\section{Conclusions}

In frail patients with supposed end-stage chronic respiratory failure that justifies treatment limitation, NIV should be attempted when acute hypercapnic respiratory failure occurs even in the case of extreme respiratory acidosis with hypercapnic coma at admission. Most of the comatous hypercapnic subjects with do-not-intubate orders survived after hospital discharge secondary to the use of NIV as a ceiling of care. The vast majority of those who survived $>6$ months were willing to receive NIV again if a new episode of acute hypercapnic respiratory failure occurred.

\section{REFERENCES}

1. Davidson C, Banham S, Elliott M, Kennedy D, Gelder C, Glossop A, et al. British Thoracic Society/Intensive Care Society Guideline for the ventilatory management of acute hypercapnic respiratory failure in adults. BMJ Open Respir Res 2016;3(1):e000133.

2. Meduri GU, Fox RC, Abou-Shala N, Leeper KV, Wunderink RG. Noninvasive mechanical ventilation via face mask in patients with acute respiratory failure who refused endotracheal intubation. Crit Care Med 1994;22(10):1584-1590.

3. Nava S, Grassi M, Fanfulla F, Domenighetti G, Carlucci A, Perren A, et al. Non-invasive ventilation in elderly patients with acute hypercapnic respiratory failure: a randomised controlled trial. Age Ageing 2011;40(4):444-450.

4. Levy M, Tanios MA, Nelson D, Short K, Senechia A, Vespia J, Hill NS. Outcomes of patients with do-not-intubate orders treated with noninvasive ventilation. Crit Care Med 2004;32(10):2002-2007.

5. Schettino G, Altobelli N, Kacmarek RM. Noninvasive positive pressure ventilation reverses acute respiratory failure in select "do-notintubate" patients. Crit Care Med 2005;33(9):1976-1982.

6. Azoulay E, Kouatchet A, Jaber S, Lambert J, Meziani F, Schmidt M, et al. Noninvasive mechanical ventilation in patients having declined tracheal intubation. Intensive Care Med 2013;39(2):292-301.

7. Clarke DE, Vaughan L, Raffin TA. Noninvasive positive pressure ventilation for patients with terminal respiratory failure: The ethical and economic costs of delaying the inevitable are too great. Am J Crit Care 1994;3(1):4-5.

8. Chu CM, Chan VL, Wong IW, Leung WS, Lin AW, Cheung KF. Noninvasive ventilation in patients with acute hypercapnic exacerbation of chronic obstructive pulmonary disease who refused endotracheal intubation. Crit Care Med 2004;32(2):372-377.

9. Fernandez R, Baigorri F, Artigas A. Noninvasive ventilation in patients with "do-not-intubate" orders: medium-term efficacy depends critically on patient selection. Intensive Care Med 2007;33(2):350354.

10. Pekmezaris R, Breuer L, Zaballero A, Wolf-Klein G, Jadoon E, D'Olimpio JT, et al. Predictors of site of death of end of life patients: the importance of specificity in advanced directives. J Palliat Med 2004;7(1):9-17.

11. Lemyze M, Mallat J, Nigeon O, Barrailler S, Pepy F, Gasan G, et al. Rescue therapy by switching to total face mask after failure of face mask-delivered noninvasive ventilation in do-not-intubate patients in acute respiratory failure. Crit Care Med 2013;41(2):481-488.

12. Kelly BJ, Matthay MA. Prevalence and severity of neurologic dysfunction in critically ill patients. Influence on need for continued mechanical ventilation. Chest 1993;104(6):1818-1824.

13. Brummel NE, Bell SP, Girard TD, Pandharipande PP, Jackson JC, Morandi A, et al. Frailty and subsequent disability and mortality among patients with critical illness. Am J Respir Crit Care Med 2017;196(1):64-72.

14. Lemyze M, Bury Q, Guiot A, Jonard M, Mohammad U, Van Grunderbeeck N, et al. Delayed but successful response to noninvasive ventilation in COPD patients with acute hypercapnic respiratory failure. Int J Chron Obstruct Pulmon Dis 2017;12:1539-1547.

15. Fletcher CM. Standardised questionnaire on respiratory symptoms: A statement prepared and approved by the MRC Committee on the Aetiology of Chronic Bronchitis (MRC breathlessness score). BMJ 1960;2:1665.

16. Charlson ME, Pompei P, Ales KL, MacKenzie CR. A new method of classifying prognostic comorbidity in longitudinal studies: Development and validation. J Chron Dis 1987;40(5):373-383.

17. Rockwood K, Song X, MacKnight C, Bergman H, Hogan DB, McDowell I, Mitnitski A. A global clinical measure of fitness and frailty in elderly people. CMAJ 2005;173(5):489-495. 


\section{DNI SubJECTs' OPINIONS OF NIV}

18. Knaus WA, Draper EA, Wagner DP, Zimmerman JE. APACHE II: A severity of disease classification. Crit Care Med 1985;13(10):818829.

19. Le Gall JR, Lemeshow S, Saulnier F. A new Simplified Acute Physiology Score (SAPS II) based on a European/North American multicenter study. JAMA 1993;270(24):2957-2963.

20. Ferreira FL, Bota DP, Bross A, Mélot C, Vincent JL. Serial evaluation of the SOFA score to predict outcome in critically ill patients. JAMA 2001;286(14):1754-1758.

21. Auzet L, Février M. Living standards and poverty in France: northern and southern departments are the worst affected by poverty and inequalities. Insee Première 2007;1162. Available at www.insee.fr/fr/statistiques/1280669. Accessed on January 17, 2017.

22. Ninot G, Soyez F, Fiocco S, Nassih K, Morin AJ, Prefaut C. The VQ11, a short health-related quality of life questionnaire for routine practice in COPD patients. Rev Mal Respir 2010;27(5):472-481.

23. Ninot G, Soyez F, Préfaut C. A short questionnaire for the assessment of quality of life in patients with chronic obstructive pulmonary disease: psychometric properties of VQ11. Health Qual Life Outcomes 2013;11:179.

24. Scala R, Naldi M, Archinucci I, Coniglio G, Nava S. Noninvasive positive pressure ventilation therapy in patients with acute exacerbation of COPD patients with varying levels of consciousness. Chest 2005;128(3):1657-1666.

25. Quon BS, Gan WQ, Sin DD. Contemporary management of acute exacerbations of COPD: a systematic review and metaanalysis. Chest 2008;133(3):756-766.

26. Díaz GG, Alcaraz AC, Talavera JC, Pérez PJ, Rodriguez AE, Cordoba FG, Hill NS. Noninvasive positive-pressure ventilation to treat hypercapnic coma secondary to respiratory failure. Chest 2005; 127(3):952-960.
27. Fox E, Landrum-McNiff K, Zhong Z, Dawson NV, Wu AW, Lynn J. Evaluation of prognostic criteria for determining hospice eligibility in patients with advanced lung, heart, or liver disease. SUPPORT Investigators. Study to Understand Prognoses and Preferences for Outcomes and Risks of Treatments JAMA 1999;282(17):1638-1645.

28. Lynn J, Ely EW, Zhong Z, McNiff KL, Dawson NV, Connors A, et al. Living and dying with chronic obstructive pulmonary disease. J Am Geriatr Soc 2000;48(5 Suppl):S91-S100.

29. Claessens MT, Lynn J, Zhong Z, Desbiens NA, Phillips RS, Wu $\mathrm{AW}$, et al. Dying with lung cancer or chronic obstructive pulmonary disease: insights from SUPPORT. Study to Understand Prognoses and Preferences for Outcomes and Risks of Treatments. J Am Geriatr Soc 2000;48(5 Suppl):S146-S153.

30. Han MK, Quibrera PM, Carretta EE, Barr RG, Bleecker ER, Bowler $\mathrm{RP}$, et al.; SPIROMICS investigators. Frequency of exacerbations in patients with chronic obstructive pulmonary disease: an analysis of the SPIROMICS cohort. Lancet Respir Med 2017;5(8):619-626.

31. Stapleton RD, Nielsen EL, Engelberg RA, Patrick DL, Curtis JR. Association of depression and life-sustaining treatment preferences in patients with COPD. Chest 2005;127(1):328-334.

32. Vincent JL. We should abandon randomized controlled trials in the intensive care unit. Crit Care Med 2010;38(10 Suppl):S534-S538.

33. Pacilli AM, Valentini I, Carbonara P, Marchetti A, Nava S. Determinants of noninvasive ventilation outcomes during an episode of acute hypercapnic respiratory failure in chronic obstructive pulmonary disease: the effects of comorbidities and causes of respiratory failure. Biomed Res Int 2014;2014:976783.

34. Lemyze M, Taufour P, Duhamel A, Temime J, Nigeon O, Vangrunderbeeck N, et al. Determinants of noninvasive ventilation success or failure in morbidly obese patients in acute respiratory failure. PLoS One 2014;9(5):e97563.

This article is approved for Continuing Respiratory Care Education credit. For information and to obtain your CRCE

(free to AARC members) visit

www.rcjournal.com

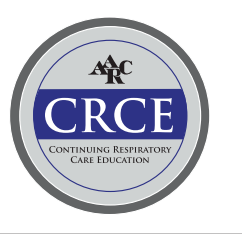

